Archives de sciences sociales des religions

188 | octobre-décembre 2019

Bulletin bibliographique

\title{
Les rites entre modernité, théologies et sciences sociales
}

Dilemmes et questions

The rites between modernity, theologies and social sciences. Dilemmas and questions

Los ritos entre modernidad, teologías y ciencias sociales. Dilemas y cuestiones

\section{Camille Tarot}

\section{(2) OpenEdition}

\section{Journals}

Édition électronique

URL : https://journals.openedition.org/assr/46704

DOI : $10.4000 /$ assr.46704

ISSN : $1777-5825$

Éditeur

Éditions de l'EHESS

Édition imprimée

Date de publication : 5 décembre 2019

Pagination : 43-71

ISBN : 9782713227844

ISSN : 0335-5985

Référence électronique

Camille Tarot, «Les rites entre modernité, théologies et sciences sociales », Archives de sciences

sociales des religions [En ligne], 188 | octobre-décembre 2019, mis en ligne le 08 janvier 2022, consulté le 07 janvier 2022. URL : http://journals.openedition.org/assr/46704 ; DOI : https://doi.org/10.4000/ assr.46704 


\section{Les rites entre modernité, théologies et sciences sociales}

\section{Dilemmes et questions}

Rites, mythes, ritologie, théologie, sciences sociales sont de bien gros mots, vastes, si redoutables que je ne les aurais pas repris dans le titre s'ils n'avaient été ceux de cette rencontre. Je vais surtout dire mes questions et mes embarras.

J'avais d'abord pensé que la journée était consacrée aux rites. Je l'aurais préféré, car j'en aurais pris un ou deux et j'aurais dit ce que j'en pense par une démarche positive ou d'analyse critique. Mais l'introduction du thème «théologie » appellerait que je me livre à un genre qui ne me plait pas, dangereux ou inutile parfois, que j'appelle le survol. Il a peut-être une utilité pour la prospection. La prospection de surface, parfois aérienne, est une pratique connue des archéologues. Mais détecter des questions n'est pas les résoudre. Survol, car j'ai découvert que dans le programme de l'année, il s'agit d'un thème encore plus vaste, puisqu'il s'agit d'interroger la notion de théologie, «objet largement fuyant », est-il dit dans l'annonce, et d'interroger ses relations, pas forcément fluides, est-il sous-entendu, avec les sciences sociales. Donc l'enjeu précis d'aujourd'hui semblerait être de savoir en quoi les rites sont-ils un bon objet pour tester les rapports des théologies et des sciences sociales.

Puisque nous n'avons pas de définition des rites, au titre d'une caractérisation première, mais provisoire et très générale, $\mathrm{j}$ ’appelle rites des actes posés par des individus, de façon répétitive ou formalisée, dans le cadre actuel ou référentiel de pratiques collectives, traditionnelles ou coutumières, parce que ces actes ou ces pratiques sont considérés obligatoires ou efficaces, pour des raisons qui restent en général implicites, mais en relation à des réalités invisibles et relatives à des croyances.

Je vais défendre l'idée que les rites ont été souvent «mal vus », dans le double sens du mot: dépréciés et peu pris en compte. Mal vus d'abord par la modernité haute ou triomphante. Ensuite, ceci remonte loin, car ils ont été parfois mal vus des théologies ou des religions elles-mêmes, surtout quand il s'agit des rites des autres. Les sciences humaines ont parfois gardé des traces de ces biais. Et certaines tentatives idéologiques de réhabilitation des rites ne rendent pas forcément service à leur compréhension sereine. 
À ma connaissance, mais je n'ai pas mené de recherches bibliographiques systématiques, il n'existe pas une ou quelques bonnes histoires qui résumeraient bien les débats sur les rites ni dans les sciences humaines, ni dans les théologies, et qui les recontextualiseraient. La matière reste à ce jour immense et dispersée.

\section{Attention, survol}

\section{Thèmes antiritualistes dans la modernité première}

Les rites prêtent facilement le flanc au soupçon d'obscurantisme. Une expérience ethnographique universelle pourrait passer pour en fournir la preuve. Quand on demande aux gens pourquoi ils font tels rites, on obtient presque toujours une réponse tautologique du genre: "parce que chez nous on fait comme ça ", " on a toujours fait comme ça ", "nos ancêtres nous ont dit de faire comme ça ». Il y a d'ailleurs là, me semble-t-il, une des raisons, non dite, pour lesquelles l'ethnographie a toujours eu plus recours aux explications holistes que la sociologie. Quand les acteurs vous disent qu'ils ne savent pas et que néanmoins ils le font, il faut bien aller chercher d'autres raisons que dans leur conscience d'acteurs pour expliquer les comportements collectifs et, en tout cas, se rabattre sur ce qui se donne et qui se laisse décrire de l'extérieur: les lieux, les temps, les corps, les gestes, les attitudes et en chercher la cohérence d'ensemble à partir de laquelle l'ethnologue relie et relit le discours des acteurs comme une partie lacunaire dans un tout et, peut-être, une rationalisation ou des constructions secondaires par rapport à des structures plus profondes dans la société, l'économie ou l'inconscient. Il semble donc que les acteurs n'ont pas besoin de connaître les raisons et encore moins les causes de ce qu'ils font rituellement pour le faire. Un discours en pointillé leur suffit. Les rites nous affrontent souvent à une catégorie essentielle de la vie sociale, qui n'a guère de place dans la pensée rationaliste: l'implicite, préférable à celle plus rude et très polysémique d'inconscient. Aussi, à partir de là, il est aisé de soupçonner les rites, les religions et les théologiens de maintenir le peuple ou le commun dans l'ignorance. Ce thème a occupé et divisé les théologies des Réformes depuis le $\mathrm{XVI}^{\mathrm{e}}$ siècle; il a été repris par la philosophie au $\mathrm{XVII}^{\mathrm{e}}$ et les Lumières, parfois les sciences sociales, se revendiquant capables d'expliquer par les causes et de libérer les individus des pratiques sans raisons. Dans un contexte de pensée rationaliste, qui fait peu de place à l'implicite, les rites apparaissent en déficit de pensée réfléchie et individualisée. Éclairer les individus revient à les inviter à les rejeter. L'émancipation remet en cause les traditions et leur emprise sur les comportements individuels.

Si les rites se reproduisent dans l'ignorance de leurs raisons, si les sujets sont peu soucieux de les chercher et si les systèmes rituels sont faits pour être répétés, il devient difficile d'exonérer les rites non seulement d'être obscurantistes, mais d'entretenir l'obscurantisme et donc la stagnation. On en tire facilement un soupçon politique: si les clergés, les théologiens se contentent de la répétition de rites dont les fidèles ne savent pas clairement les raisons, c'est qu'ils y ont 
un intérêt, généralement de pouvoir. Les rites médiatisent la domination qu'ils relaient, instillent l'obéissance, soumettent à l'autorité, imposent l'immobilisme. Ils sont en effet plus conservateurs que progressistes.

Associés à des pratiques collectives, ils sont soupçonnés par les individualistes de normer les individus selon des contraintes toutes extérieures et arbitraires, d'imposer les conformismes, de raboter la singularité des âmes et des corps. Maints rites ont en effet, comme dans les rites de passage, une évidente dimension disciplinaire. Les rites symbolisent alors l'extériorité du social qui fait irruption dans l'intériorité. Ils seraient intrusifs, enfermant, aliénant. Une société qui émancipe l'individu l'émancipe des rites, qui sont des contraintes collectives, sinon des ingérences.

Même si beaucoup de rites offrent des similitudes troublantes, comme les sacrifices ou certains rites de passage, même si on ne connaît guère de société sans rites, il est facile de trouver dans l'abondance des variations rituelles la marque et le bastion des particularismes, et donc de la division. L'histoire est pleine de querelles de sacristie qui ont dégénéré en schismes, voire en guerres de religion. D'autant plus que les ritualistes des diverses religions ont une évidente tendance à voir plutôt ce qui sépare leurs rites de ceux des autres et donc à dénier leurs ressemblances. Des simples fidèles aux rubricistes les plus sourcilleux, l'histoire des religions met devant la foule de ces dévots exacts, pointilleux sinon obsessionnels, qui ont le génie de maximaliser les différences, de les exacerber et parfois d'en faire des motifs de conflit. Ce qui renforce le fixisme, quand toute variation devient un danger qui met en cause la légitimité et l'efficacité $\mathrm{du}$ rituel. Les rites tombent facilement sous l'objection universaliste de diviser les hommes, de les enfermer, voire de les opposer arbitrairement ou pour des futilités. Les valeurs universelles passent donc par le dépérissement des rites.

Beaucoup d'autres objections contre les rites ont été tirées de leurs formes et de leurs contenus. Pour la forme, ils sont liés à l'imitation et à la répétition. L'objection de formalisme est une des plus récurrentes: asservissement à des formes vides de sens ou d'âme, non comprises, usées par la répétition, stérilisant l'invention et la créativité, la spontanéité du vécu. Les rites sont un domaine où règnent les modes de pensée analogiques. Beaucoup de rites choquent par des associations d'idées imprévues. D'où l'objection logique, contre une pensée analogique, métaphorique, peu rigoureuse et la construction des rites en bastion de la pensée prélogique, de la mentalité primitive. Les contenus réfèrent à des croyances souvent exprimées dans des mythes qui contredisent aussi la rationalité. Ils renvoient à de l'impossible, du miraculeux, et ils choquent souvent la morale ou la justice. L'objection morale et moralisante accompagne la critique des rites depuis les réformes protestantes et catholiques, souvent suivies sur ce point par les Lumières. Il est difficile de trouver une page de Frazer où il ne s'indigne pas.

Si maintenant on les classe parmi les pratiques, c'est qu'ils relèvent bien de l'action de l'homme sur l'homme. Mais il est facile de les associer à des manipulations, voire à des conditionnements, ou à des agressions contre le corps, le psychisme, des atteintes à l'intégrité de l'individu. 
Avec l'essor de la pensée technicienne et utilitariste depuis le milieu du $\mathrm{XVIII}^{\mathrm{e}}$ et tout au long du XIX ${ }^{\mathrm{e}}$ siècle, l'objection dominante porte sur l'efficacité et l'utilité. Et s'ils ne sont pas techniquement efficaces, sont-ils quand même fonctionnels à un autre niveau, social ou psychique, conscient ou inconscient? Plus se développe, depuis l'Encyclopédie et pendant tout le $\mathrm{XIX}^{\mathrm{e}}$ siècle, la réflexion sur les technologies, plus les rites en sont séparés, plus leur association à des techniques parait incompréhensible ou superfétatoire. Or la modernité a été mue par la foi en la technologie, par l'évidence de son progrès cumulatif. Si beaucoup de rites mettent en œuvre des techniques, ou offrent une analogie avec elles, ils les entourent aussi comme une gangue. On a souvent jugé cette association incongrue, inutile ou incompréhensible. Cet étonnement sous-tend aussi l'enquête de Frazer. De là à les rendre responsables de la stagnation technique des sociétés traditionnelles, le pas a été souvent franchi.

Éloignés de la technologie, les rites ont donc été toujours plus associés à la religion et à la magie, ce qui ne plaidait pas en leur faveur. Ils ont donc été pris dans toutes les fluctuations de la critique de la magie et de la religion. Mais cette tentative de confinement dans la religion révèle sa limite en posant un problème toujours irrésolu: tous les rites sont-ils religieux ou que religieux? Une société athée est-elle entièrement sortie des rites, des seuls rites religieux ou de tous les rites et jusqu'où?

L'enquête est à mener: Lévy-Bruhl, Frazer, mais bien avant Hegel ou Spinoza, ou les grands conflits autour des Réformes depuis la fin du Moyen Âge. L'enjeu serait de voir la place des rites dans la genèse de thèmes qui, en se transformant, passeront des Réformes aux évolutionnismes: critique des traditions, de l'ignorance, responsabilité des clergés, illégitimité des savoirs populaires, accusation de magie, nécessité de rectifier la pensée commune par les savoirs lettrés puis savants. Il semble bien probable que la critique des rites a joué un rôle dans la genèse des réformismes religieux, puis du progressisme culturel et politique et enfin de l'évolutionnisme scientifique en sociologie et en anthropologie. Les rites ont été une des bêtes noires des progressismes et les tentatives de réévaluation par des courants plus conservateurs, comme le romantisme au XIX $X^{\mathrm{e}}$ siècle n'ont pas forcément rendu service à leur compréhension. J'y reviendrai à propos d'Eliade. Dans cet héritage, on a vu le succès depuis Woodstock ou Burning Man des grands rassemblements festifs et le questionnement sur leur composante rituelle, bien que nullement confessionnelle. Avec les courants contre-culturels américains des années 1960, on voit des Occidentaux, après Katmandou, continuer d' aller sur les marges par exemple amazoniennes (Amselle, 2013) pour reconstituer - sur les doubles débris du rationalisme auquel ils ne croient plus et des cultures indigènes mises en morceaux par la colonisation et la christianisation - sinon les rites, du moins des pratiques, en particulier thérapeutiques ou addictives, que leurs prédécesseurs occidentaux n'avaient eu de cesse de combattre ou d'éradiquer chez eux et chez les autres, souvent au nom de la vraie foi, de la raison, de l'émancipation ou encore du progrès. Il manque pour les qualifier de rites la continuité d'une tradition, mais ça n'empêche pas de la réinventer. 
Ce sont sûrement des néoritualités, nées en double discontinuité, mais en réaction contre cette discontinuité, puisque ce sont souvent des Occidentaux qui réapprennent le chamanisme, qui ne leur était pas autochtone, aux Indiens pour qui il ne l'est plus tout à fait.

Mais ceci montre que peut-être davantage que des franges significatives de l'Occident sont en train de passer ici d'une critique des rites et des traditions au nom du rationalisme et/ou de l'individu à émanciper, à la revendication par la liberté individuelle de se libérer du rationalisme et de se composer le cocktail de pratiques choisies dont l'individu juge avoir besoin pour répondre à sa demande de sens ou de bonheur. Un tour est joué dans cette affaire et une boucle est bouclée. Le discours sur l'aliénation par les rites et les coutumes s'est inversé, précisément parce que les traditions ont été rompues et que, n'étant plus vécues, elles en sont que plus facilement désirées ou phantasmées. Les pratiques sont sorties des religions, du social et des pouvoirs qui sont déniés ou occultés, mais elles sont référées au primitif, au sujet individuel et à la notion extensive de spiritualité, justifiée par une science du cerveau ou des sécrétions physiologiques qui en montre les bienfaits pour l'individu. Peut-être parce qu'il défend des positions rationalistes (et donc se situe dans la continuité d'une tradition de pensée), Amselle a relevé cette inversion (que j'appelle dans mon jargon "pharmakologique ", car il s'agit de savoir si le rationalisme est un remède, position classique depuis le XVII ${ }^{\mathrm{e}}$ siècle - et même avant, avec Aristote ou Thomas d'Aquin - ou un poison, position qui prend le dessus dans les courants romantiques):

Quel anthropologue oserait désormais prétendre que tel magicien, marabout, gourou ou chamane s'ingénie a priori à tromper le peuple, que la transe chamanique ou autres est un instrument d'aliénation? On insistera, à l'inverse, sur le caractère symbolique à l'œuvre dans la divination, la magie ou le traitement délivré par le traditipraticien et sur l'action de ce dernier comme donateur de sens. Cette idéalisation de la magie et de la religion par l'anthropologie actuelle, notamment amazoniste, idéalisation qui trouve son climax avec le cognitivisme, risque de faire bon marché de ce qui avait été pointé par les Lumières à propos du chamanisme, à savoir que cette institution est aussi une technique de pouvoir. C'est en abstrayant les conditions pratiques d'exercice du pouvoir des magiciens, des devins ou des marabouts au sein de leurs communautés respectives qu'on a pu en faire un mode de connaissance désincarnée, une pensée mythique ou «sauvage» intemporelle (Amselle, 2013: 220).

Il faut ici interroger la coupure majeure et symptomatique de ces faits, toujours revendiquée, entre religion, renvoyée du côté du négatif, du collectif, du particulier, de l'oppressif et spiritualité, revendiquée comme le positif, universel, mais strictement individuel. Cette disjonction du bonheur de l'individu et du politique sous toutes ses formes est une des caractéristiques de cette tendance que j'appelle «néo-gnostique». La grande différence avec le gnosticisme ancien est qu'il n'est pas dualiste et qu'il est souvent matérialiste: il refuse la dualité âme-corps, il inclut le corps dans la singularité, mais il sépare totalement le bonheur de l'individu ou son éveil, la connaissance de soi 
de celle des médiations sociales. Ce n'est pas le rite qui fait l'individu comme dans les sociétés traditionnelles, c'est l'individu qui fait les rites qu'il se choisit. Il est bien l'héritier du romantisme dont il combine deux traits : maintien du principe moderne du primat de l'individu, mais en réaction contre la modernité rationaliste (besoin de rites et d'expérience religieuse puisée ici ou là).

\section{Les rites, les conflits théologiques et la division des chrétiens}

Avant de diviser la modernité, les rites sont un sujet qui divisa les théologiens et plus gravement les chrétiens, les Églises et les sectes et parfois les religions entre elles.

Les questions rituelles sont au cœur des conflits du christianisme naissant avec le judaïsme: place du Temple et des sacrifices sanglants, respect du sabbat, et sans doute le débat le plus fondamental autour de la circoncision. Pour les chrétiens les sources ne manquent pas. Les évangiles s'inscrivent dans des traditions prophétiques de critique de la pensée sacrificielle, des abus des rites et du Temple de Jérusalem. Puis le paulinisme, première synthèse savante des spécificités chrétiennes, fournit un argumentaire fondamental pour la suite autour de quelques thèmes: le salut par la foi plus que par la loi, la dialectique plus large de la lettre et de l'esprit. Les tensions sur la ritualité en christianisme se concentrent donc sur les héritages d'une tradition prophétique venant du judaïsme et retournée contre lui, entre rejet et intériorisation. Car la vie spirituelle demande aussi l'intériorisation des rites, au moins de leur vocabulaire (par exemple dans le thème paulinien du sacrifice spirituel). Puis le christianisme des premiers siècles reprend la critique juive du paganisme et de son système sacrificiel au cœur de la vie des cités. Il accentue ce mouvement de spiritualisation déritualisante en se présentant non seulement comme la vraie religion mais comme la vraie philosophie accomplie, reprenant certaines critiques philosophiques de la religion des cités comme des superstitions populaires. Les tensions sont vives et la voie étroite entre les exigences du culte en esprit et vérité et les tentations d'un spiritualisme exalté, gnostique, mais qui entre en conflit avec les contraintes de la communication de masse pour une Église de plus en plus multitudiniste et accueillante à des habitudes païennes jugées superstitieuses. Ces tensions se retrouveront dans le monachisme.

Dans la tradition orthodoxe, la liturgie, donc le rite, véritable théâtre sacré, restera le sol même de la théologie, sa base et en un sens son terme: la theoria y garde son sens premier de savoir contemplatif, dont le but est de voir ce dont la parole ne parle qu'en son absence. Ce fut sans doute la même attitude dans la théologie catholique jusqu'au XIII ${ }^{\mathrm{e}}$ siècle, et ça le reste dans une certaine théologie monastique ou spirituelle pendant le Moyen Âge et jusqu'au XVII ${ }^{\mathrm{e}}$ siècle avec les mystiques, jusqu'à leur crépuscule. Mais, une autre rupture apparaît, avec le projet d'une théologie comme science, qui a son site dans les écoles et à l'université, qui change le sens de la theoria chrétienne, devenue plus intellectualisée, moins contemplative et déritualisée. 
Cette rupture prépare la troisième, violemment antiscolastique, avec le protestantisme qui réveille le prophétisme initial sous la forme d'un paulinisme retourné contre l'institution catholique: le salut par la foi seule est brandi contre les œuvres assimilées aux pratiques rituelles, il conteste donc le monopole des clercs sur la ritualité et il induit un élagage drastique des sacrements qui passent de sept à deux, vidant la liturgie des sacramentaux et la recentrant sur le sacrement de la Parole.

Une quatrième rupture tient aux effets de la Réforme protestante sur la Réforme catholique, qui reprend l'épuration des rituels, lutte contre les superstitions populaires (cf. l'abbé Thiers). La création d'une "religion populaire ", bastion de pratiques coutumières mais devenues suspectes, à réformer selon les critères orthodoxes et savants, commence à cette époque de la Contre-Réforme et ressurgit jusque dans les sciences sociales du $\mathrm{XX}^{\mathrm{e}}$ siècle, quoique dans une tout autre problématique.

Mais le point commun à ces deux courants, l'élaboration de la théologie comme science et la réforme des pratiques populaires, est le processus d'intellectualisation, de spiritualisation et d'individualisation du religieux, impulsé par des clercs qui se présentent comme des savants, des litterati, comme le montre le projet né en protestantisme d'une alphabétisation généralisée pour pouvoir accéder de fait au Livre du salut que l'imprimerie rend disponible. Dans l'ère chrétienne occidentale l'alphabétisation a accompagné la déritualisation. Mais ailleurs?

Le processus se radicalise par des scissions dans les milieux des cléricatures lettrées: multiplication des écoles de pensée à la fin du Moyen Âge, puis des courants, divisions entre les clercs (le protestantisme comme "hérésie intellectuelle»), les conflits entre jansénistes et jésuites, en attendant celui entre Philosophes et clergé. Avec la diffusion des livres et la presse, c'est l'engendrement de l'opinion publique au sens moderne, cette nouveauté qui échappe à l'emprise des ritualités traditionnelles. L'enjeu n'est plus la seule réforme de la religion, mais la prise de contrôle de la culture et de l'opinion publique naissante, domaine qui ne cesse de s'élargir au débat sur les types d'hommes qu'il s'agit de produire.

La question des rites a été au cœur des conflits confessionnels chrétiens modernes. Le réformisme chrétien divisé par la question rituelle a préparé le sol du réformisme laique et scientifique. Il indique les objections antirituelles: l'inutilité d'un faux culte, la corruption des sacrements, la prolifération inutile des pratiques, les abus des clergés, l'inutilité des virtuoses, le poids paralysant des traditions. Il indique l'enjeu, le salut de l'individu et l'issue: l'intériorisation $\mathrm{du}$ religieux comme spiritualisation et son individualisation. Il a engagé les efforts pour sortir des conflits confessionnels par la science qui se laïcisera, et donc par la science des religions. Il a ouvert la voie aux processus dont les Lumières et la modernité feront une sécularisation.

Les conflits autour des rites se retrouvent dans toutes les religions surtout naissantes. On pourrait prendre l'exemple du bouddhisme. Les fortes tendances antirituelles et antiritualistes du bouddhisme ancien, tournées contre le 
brahmanisme, n'ont pas empêché la prolifération rituelle des bouddhismes tantrique et tibétain. Singulière revanche. Ou l'islam naissant. Le conflit du Prophète avec Quraysh commence autour du culte rendu dans l'enceinte sacrée de la Mecque. Il continue par le choix de la prière tournée vers Jérusalem, puis avec l'hégire, retournée vers la Mecque, puis avec la reconquête, par la purification du sanctuaire et l'instauration du culte musulman, avant son extension à l'Arabie.

\section{Rites et sciences sociales, héritages et apories}

Dans quelle mesure nos sciences sociales ne sont-elles pas marquées, à travers les Lumières, par ces héritages des confessions en tension, sur des grands thèmes débattus: traditionalisme, ritualisme, formalisme, domination, aliénation, conservatisme, progressisme, etc? Une archéologie croit pouvoir détecter des sensibilités confessionnelles rémanentes.

La question des rituels pose donc celle des choix d'objet, de la neutralité axiologique et de la critique épistémologique à mettre en œuvre. D'autant plus qu'après un bon siècle de travail les sciences sociales se heurtent toujours à trois dilemmes, trois apories: la relation entre mythe et rite, et donc l'importance relative des rites, le rapport entre rites et religions, enfin entre rites et sacrifices. Je vais dire un mot des affinités électives puis des apories.

\section{La question des affinités électives}

Y a-t-il des présupposés confessionnels au moins sous forme de sensibilité culturelle ou d' "affinités électives » dans l'approche des problèmes rituels par les sociologues ou les historiens?

Ça me semble un chapitre possible dans le livre un peu ressassé des différences entre Durkheim et Weber, dont il faut faire autre chose qu'une nouvelle querelle scolastique entre Platon et Aristote! Il me semble que la thèse durkheimienne la moins facile à admettre, à savoir que la religion aurait été la première des institutions, se fonde ou tombe selon la place qu'on reconnaît aux rituels dans l'archéologie des sociétés humaines. Mauss était conscient que les rites sont la forme élémentaire de l'institutionnalisation dans les sociétés sans écriture, et même encore très longtemps après l'apparition de l'écriture, et qu'ils sont donc au centre de la sociologie, surtout de la religion, si la sociologie comme il le prétendait est l'étude des phénomènes d'institution, dont un des modèles pour lui était la langue.

Or on ne voit ni une place analogue pour le rite chez Weber, ni l'esquisse d'une théorie construite du rite comme telle chez lui. Un ouvrage sous la direction de Hans Kippenberg et Martin Riesebrodt (2001) a tenté de systématiser l'autre sociologie de la religion de Weber, non pas le rapport des religions universelles à l'économie, mais ses idées sur le phénomène religieux en général. En voici les têtes de chapitre : Le hiatus irrationalis, la rupture entre l'être et le devoir être. La religiosité. Le développement religieux. Les communautarisations 
religieuses. La Magie, l'enchantement et le désenchantement. Le Polythéisme. Le Charisme. Le Prophète, le prêtre et le virtuose. La prophétie éthique et exemplaire. Les religions de salut. Le refus du monde. La mystique. L'ascèse. Le ressentiment et les parias. L'intellectualisme. L'antagonisme des valeurs.

On a donc bien là les grandes catégories transversales qui font la richesse et l'originalité de la sociologie générale de la religion de Weber. Mais on remarquera qu'il n'y a pas de rubrique « rite» ni d'ailleurs «mythe », ni «sacré ». L'Index du volume ne contient qu'un seul renvoi aux rites, à propos des cultes helléniques classiques, du pluralisme rituel rendu à des dieux en lutte les uns avec les autres (Kippenberg, Riesebrodt, 2001: 131). Je dirai qu'il ne faut pas commencer par s'en plaindre. C'est sans doute à cette absence d'une catégorie trop classique dans le discours sociologique, et d'ailleurs héritée du langage religieux lui-même, que l'on doit une partie de la prodigieuse fécondité, de l'audace intellectuelle de la sociologie wébérienne. Cette absence des catégories trop indigènes au fait religieux et trop traditionnelles du mythe et du rite dans la sociologie wébérienne atteste donc de sa nouveauté. Ne commençons pas par la prendre comme un manque, alors que c'est probablement le prix d'une puissante originalité et d'un déplacement dû à l'individualisme méthodologique. En ne faisant pas un sort spécial à cette catégorie, Weber s'est éloigné des sentiers battus et des holismes hérités, pour mieux penser la modernité religieuse et proposer des catégories inédites. Sa sociologie est partie de phénomènes plus typiquement contemporains, prenant acte que dans les sociétés contemporaines et individualistes le rite religieux n'a plus l'importance de jadis. Weber part du protestantisme et de la modernité, il remonte vers l'archaïque du religieux, mais ne va pas jusqu'à retrouver le rite comme un objet à constituer en catégorie propre. Ce constat pose la question de l'affinité élective de la sociologie de Weber avec le protestantisme d'une part et la modernité de l'autre, du fait du rapport étroit entre modernité et protestantisme. Pour parler comme les scolastiques, la modernité et le protestantisme sont très loin de fournir tout l'objet matériel de sa sociologie des religions, puisqu'il parle tout autant des autres grandes religions universelles. Mais ils lui fournissent son objet formel, son point de vue et sa problématique forgée à partir de la relation de la modernité, du protestantisme, du capitalisme et de l'individualisme, puis étendue aux autres religions. Le holisme durkheimien s'adosse, au contraire, à une conception objective du sacré, inévitablement appuyé à des ritualités. Ses références premières viennent de deux systèmes rituels particulièrement puissants, mais plus traditionnels, antérieurs à la modernité, le judaïsme et le catholicisme.

Une fois qu'on a rendu justice à la nouveauté de Weber, ce fait nous met devant une tâche. Si le rite n'est pas une des grandes catégories transversales de la sociologie wébérienne de la religion, Weber apporte néanmoins beaucoup pour le penser, mais à condition de remembrer ses vues. Il faut aller chercher l'objet souvent en creux, car il est présent dans la magie, dans les religions de salut, dans les pratiques de l'ascèse, il borde le charisme, etc. Les problèmes rituels sont là sous-jacents mais non dégagés pour eux-mêmes. On voit qu'ils sont présents dans les conflits du prophète, du prêtre et du virtuose, conflits internes 
au religieux, mais qui mettent en cause le statut des rituels. Or ces conflits ne sont pas sans recoupement, non plus, avec la fameuse trilogie de la Herrschaft: pouvoir patrimonial, pouvoir charismatique, pouvoir bureaucratique rationnel. Car j'essaierai de défendre la thèse qu'un rapport au rituel, non traité par Weber, joue dans leurs différences. La domination patrimoniale repose sur des traditions, dont plus ou moins de rituels. La domination charismatique suppose soit la rupture des rituels reçus, le constat de leur obsolescence, soit la mise en cause de leur légitimité. La personnalité charismatique dit: « ne suivez pas l'ordre effondré, mais suivez-moi et faites comme je fais». Mais elle réussit si elle impose son ordre. Revanche du rituel sur le charisme, la mal traduite routinisation du charisme est largement contenue dans les conditions de possibilité, puis de succès, du charisme. L'énigme impensée de la trilogie, c'est le rapport de la domination rationnelle-bureaucratique avec le rituel. Weber est silencieux sur ce point. Il voit bien que la bureaucratie est un phénomène très ancien qui s'est imposé en Mésopotamie, Égypte, Chine et qu'on suit dans toute l'histoire occidentale avec l'Empire romain, l'Église médiévale, l'État moderne. En même temps, nul doute que pour lui l'extension de la bureaucratie est un des faits majeurs des sociétés les plus modernes et industrialisées.

Le rôle des rites reste à remembrer et à expliciter, car jamais il ne passe sur le devant de la scène wébérienne. Il reste dans l'impensé de sa sociologie. C'est sans doute une conséquence de son individualisme. Mais l'hypothèse que j'essaierai de défendre est que la voie proprement wébérienne que sa sociologie offre pour penser les rituels, même s'il ne l'a nulle part systématisée, c'est le passage chez lui de la rationalité au pluriel. Weber voit bien que les systèmes de cultes ont opéré des formes de rationalisation de la conduite sous le double aspect de la rationalité parfois instrumentale, plus souvent axiologique. Mais la même chose vaut de formes rituelles qui peuvent nous paraître étonnantes, ou choquantes, comme certaines formes d'ascèses, de renoncement au monde, etc. Pour mesurer ce que signifie ce passage d'un rationalisme moniste de la Raison au rationalisme éclaté des rationalisations multiples que Weber décrit, il faudrait comparer ces deux contemporains qui ne vivent déjà plus dans le même monde intellectuel: Frazer et Weber. Weber a rompu avec la philosophie unitaire et téléologique du rationalisme, où une forme unique de rationalité pourrait recueillir et réunifier la diversité multiple des rationalisations en tous genres et dans les divers ordres mis en œuvre par les individus et les groupes.

La comparaison Weber-Durkheim impose la tâche de réfléchir à ce que j'appelle le "grand écart». Le grand écart, c'est la situation inconfortable de la sociologie, de l'ethnologie ou de l'anthropologie quand manifestement l'évolutionnisme devient impossible. J'entends ici une philosophie de l'histoire de la culture (qu'elle suppose ou non un évolutionnisme biologique, car elle a commencé avec les premières formulations du progrès, un bon siècle avant l'évolutionnisme en biologie), marquée par trois traits: 1) ce que Lenclud a appelé le "grand partage", cette grande coupure, entre nous et les autres, c'est-à-dire les civilisés et les sauvages; 2) le progrès orienté comme une 
flèche garanti par une loi de la nature, de la vie ou de l'âme ou de la raison; 3) l'inconditionnelle supériorité de l'Occident.

La grande coupure n'est pas justifiée parce qu'elle postule que l'archaïsme mental, phantasmatique, comportemental ou axiologique serait tout entier du côté des autres, les primitifs, alors que tout serait neuf ou moderne ou contemporain chez les civilisés. Or, en réalité, l'archaïsme est aussi une catégorie de la «civilisation", et les sociétés traditionnelles sont aussi contemporaines. Deuxièmement, la conception téléologique dans le domaine historique, sous forme de la croyance au progrès, est aussi scientifiquement injustifiable que dans d'autres domaines. De plus la croyance au progrès dans ce qu'elle avait d'apparemment de plus solide, le progrès technique et scientifique, vient d'une mauvaise interprétation d'un fait réel: le caractère cumulatif de la technologie et de la science. La supériorité de l'Occident est un jugement de valeur fondé sur un ethnocentrisme conforté par une situation historique passagère de domination qui ne reposait sur aucune essence, mais sur des rapports de force dans une conjoncture historique qui devient chaque jour plus questionnable.

Mais si la critique de l'évolutionnisme n'est plus à faire, elle nous laisse cependant devant des difficultés considérables que j'appelle le "grand écart ». Il est quand même difficile de nier que nos sociétés se transforment vite et profondément et s'éloignent chaque jour des sociétés traditionnelles, beaucoup plus vite que les continents ne se séparent; que les petites sociétés chères aux ethnologues se font rares et même rarissimes et semblent partout menacées d'ethnocide, que certaines technologies se mondialisent à une vitesse étonnante, etc. Si la dérive des continents n'est pas un progrès, leur éloignement est quand même un fait. Le grand écart est la tâche où nous sommes de penser ces différences entre des univers culturels qui, se différenciant toujours plus, exigent toujours un plus grand détour, alors qu'on ne peut plus compter sur une solution toute faite et paresseuse comme le progrès pour fixer les différences.

Or, il me semble que l'anthropologie des religions est traversée et donc concernée au premier chef par le grand écart, visible dans tout ce qui sépare une approche du religieux par les formes contemporaines, et donc à dominante individualiste, et une approche par l'histoire et l'histoire comparée, l'ethnologie, les archéologies qui livrent des traces de formes plus traditionnelles, plus holistes, et où le rôle des individualités devient plus difficile à saisir. C'est la difficulté où nous sommes de faire une socio-anthropologie qui rapproche les deux bouts, le proche et le lointain, quand on ne peut plus, pour les réunir, recourir à des présupposés évolutionnistes: simplicité primitive des formes anciennes, concentration de l'archaïsme dans l'altérité, supériorité des formes modernes sur les formes anciennes, évolution programmée et garantie par une téléologie, loi univoque de développement ou d'évolution, récapitulation de l'histoire dans le présent, ethnocentrisme occidental qui pose la supériorité de ses propres formes en tout domaine, etc. Surmonter le grand écart supposerait de résoudre trois apories tenaces. Or elles concernent les rituels. 
54 - ArChives de SCIENCES SOCIALES DES RELIGIONS

\section{Trois apories}

\section{L'aporie rite et mythe}

Dilemme classique de la poule et de l'œuf. Posé en termes trop généraux, le problème se retourne toujours. Donc on soupçonne qu'il n'y a plus grandchose à en attendre. Ce n'est pas mon point de vue, et je crois que c'est une question difficilement évitable.

On peut donner des exemples: les hésitations de Durkheim qui finit par donner la priorité au mythe et donc aux représentations. Mauss, au contraire, fut le grand ritologue, il devait grandement aux hindous l'idée qu'au commencement du social humain est l'acte rituel. Mais à la fin de sa vie, comme pris d'un remords, il s'inquiète de leur avoir peut-être trop sacrifié, ce qui donnera bonne conscience à Lévi-Strauss pour privilégier quasi exclusivement l'étude des mythes.

Largement dans la suite de Mannhardt, Eliade semble réévaluer pleinement le rite. Mais sa fausse belle synthèse parait une voie de garage, une synthèse abstraite qui néglige ce qui la contredit. Il pose un rapport en miroir quasiment parfait et constant du mythe comme programme et du rite comme son actualisation et, comme le mythe parlerait exclusivement de l'origine (ce qui est faux), le rite serait un retour à l'origine. Comme si les mythes étaient des livrets d'opéra qui ne parlent que de naissance du monde, que le rite joue et actualise. Cette harmonie d'un rapport circulaire et sans faille de la théorie et de la pratique caractériserait la religion sauvage archaïque et prouverait sa capacité à surmonter l'histoire, ce qui est faux, car quand ces sociétés rencontrent une historicité chaude, elles sont immédiatement menacées. C'est une construction idéologique, qui transfère sur un néopaganisme, comme répétition dans un monde plein comme un œuf primordial, une nostalgie orthodoxe d'un cosmos sacré. Mais le rapport n'est pas en miroir. Les mythes parlent de tout et pas seulement d'origine, on ne peut réduire le discours mythique au mythe d'origine, non plus que les pratiques rituelles à la relation à l'origine.

Avec beaucoup d'autres, Lévi-Strauss a bien vu qu'il y a des mythes sans rites et des rites sans mythes. Mais il est allé si loin en sens inverse d'Eliade pour dissocier le mythe et le rite, qu'il a complètement autonomisé le mythe en négligeant les rites. Le mythe " objet absolu » comme l'œuvre d'art moderne, est un pur produit de l'entendement. L'intellectualisme lévi-straussien explique le mythe par les structures et le cerveau, mais avec peu de relation aux pratiques sociales. Non seulement on sort de la sociologie, mais est-il certain que les rites ne sont pour rien dans les processus d'intellectualisation et de catégorisation: n’y jouent-ils pas un rôle par la répétition, la formalisation, les séquençages, la schématisation? Dans la mesure où maintes formes de théâtre sont la continuation de rites, ne sont-elles pas la preuve de ce rôle?

L'intellectualisme et le formalisme ont leur droit comme dans le structuralisme naguère ou le cognitivisme qui ont donné la préséance aux mythes. Mais je crois que les sociologues doivent donner la prééminence aux 
rites, s'ils veulent échapper aux pièges de l'idéalisme et chercher une approche réaliste. Car la société, les liens sociaux se construisent certes par des opérations intellectuelles, mais liées, attachées à des rapports de force. Enfin, peut-on totalement séparer la forme et le contenu dans les rites? N'y a-t-il aucune pertinence dans la critique de Girard? Le mythe et le rite ne se contentent pas de distinguer, ils mettent en forme des forces et en refoulent d'autres. Ils mettent à distance. Ce qui pose la question des rapports du rite, de la religion et du sacrifice.

\section{Rites et religion}

Dans la culture courante, et surtout dans la langue aujourd'hui encore, le mot «rite" reste comme un syntagme immédiatement relié au religieux. Comme si les deux mots s'appelaient par une relation essentielle ou logique ou un monopole. La philologie comparée du latin et du sanscrit montre l'ancienneté du mot rite, qui remonte à l'indo-européen commun. Elle montre aussi que la notion de rite est nettement plus ancienne que celle de religion, plus récente et latine. Or aujourd'hui, du fait de la sécularisation, la sociologie contemporaine a de plus en plus tendance à séparer les rites et le phénomène religieux. Dans la crainte de ne pas recueillir comme tels des rites qui ne sont pas associés aux religions classiques, elle a tendance à parler, comme Rivière, de « rites profanes". Mais on sait aussi que cet usage ne va pas sans un risque d'oxymore ou de dilution. Car si on sort la catégorie de rite du religieux, ne va-t-on pas la dissoudre en appelant rite toute pratique collective répétitive et formalisée? Et donc voir du rite partout, même là où il n'y en a pas? Les relations du rite et du religieux sont donc toujours débattues, loin de les simplifier, les phénomènes de sécularisation les ont en fait compliquées.

Comme les religions, les rites semblent bien investir et parfois structurer trois domaines, à partir desquels on cherche d'ailleurs à les penser. Le premier est le domaine mythico-théologique qui renvoie aux récits et aux expériences concernant des invisibles. C'est d'ailleurs à partir de ce domaine qu'on a défini la religion comme relation aux dieux et qu'on privilégie les discours sur les divinités comme le spécifique du religieux. Selon une définition classique mais étroite de la religion, on définit les rites par la mise en œuvre de cette relation. C'est le point de vue des définitions religieuses traditionnelles du rite. Mais les religions ont aussi un rapport avec la nature et la cosmologie. On soulignera que les rites cadrent des actions humaines dans un espace-temps qui leur est propre, mais qui est différent de l'espace-temps des mythes, car il est cadré dans un ordre global du monde dans lequel les rites jouent un rôle. Enfin, les religions et les rites jouent un rôle dans un troisième champ, socioanthropologique, où il s'agit d'instaurer, contrôler, réparer, reproduire la société ou de la restaurer, mais aussi d'instituer et de socialiser les individus (comme dans les rites de passage) et donc de gérer l'articulation des individus qui n'est pas entièrement donnée, mais toujours à reconstruire. 
Ces trois champs qu'articule le religieux définissent aussi trois niveaux d'analyse et de compréhension des rites: théologique voire phénoménologie de la conscience pratiquante et croyante, cosmologique ou par exemple aujourd'hui des approches éthologiques et d'éthologie comparée, enfin socio-anthropologique. Je ne pense pas qu'on puisse aller beaucoup plus loin que la juxtaposition de ces trois niveaux d'approches si on n'affronte pas la troisième aporie.

\section{Rites et sacrifices}

C'est le débat le plus difficile, dans les sciences sociales des religions, celui que nous avons tendance à refouler dans l'archaïque ou l'exotique, le sacrifice n'étant plus une catégorie institutionnellement reconnue de nos sociétés. Pire, c'est un objet politiquement incorrect. On en a tellement accusé ses ennemis, on veut tellement en protéger ses amis et soi-même. Un objet encombrant, comme qui dirait la pomme de terre chaude des sciences des religions promptes à s'en défaire. Aujourd'hui, on se méfie pour de bonnes raisons des théories unitaires du sacrifice. D'où un conflit d'école dans l'opposition frontale entre Lévi-Strauss, pour qui le sacrifice parait à la limite du bon sens, et Girard. Je crois que le sacrifice est au cœur de l'inconfort de cette position que j'ai appelée le "grand écart». Le sacrifice revient comme l'objet sans doute le plus gênant du grand écart: les "progressistes » n'avaient pas de peine à en faire une folie des sociétés archaïques, les "primitivistes » veulent en exempter les sociétés les plus traditionnelles qu'ils aiment et en accuseraient plutôt les plus développées; les "humanistes" veulent en faire une exception malheureuse, etc.

Peut-être ai-je mal lu, mais j'ai cru me rendre compte que Jean-Pierre Albert connaissait aussi ce grand écart. Il me semble que la question se pose si on rapproche l'article «Rite» (Albert, Piette, 2010) et le livre collectif qu'il a dirigé sur le Sacrifice humain en Égypte ancienne et ailleurs (Albert, 2005). Ces deux travaux m'intéressent autant l'un que l'autre, parce qu'ils posent des problèmes que je me pose tout autant. Néanmoins ils sont différents et illustreraient une posture d'inconfort qui n'apparaît pas si on ne les rapproche pas, mais qui me parait celle de notre discipline. L'article «Rite» est de sociologie pure. Il ne parle que du contemporain. La méthode est purement synchronique. Le rite est étudié à partir de la contemporanéité d'une société sécularisée. Il fait très peu d'allusions au contenu des rites. La perspective est formaliste et cognitiviste. La question du rite peut se traiter sans aucune allusion à quelque sacrifice que ce soit. Il en va tout autrement dans le livre qui fait dialoguer des historiens, surtout des archéologues et quelques ethnologues. La part du lion, la moitié du livre revient à l'Égypte prédynastique. Bien que la question ait longtemps passé pour impossible à poser, taboue, pour une civilisation aussi remarquable, aussi raffinée, j'allais dire aussi amie, il faut s'y résigner: au début du système pharaonique, il a pu se produire des sacrifices humains. Il me semble que le livre confirme que le débat autour du rapport du rite à la religion est suspendu à la définition du sacrifice. 
Je crois qu'on peut le suggérer rapidement à partir de la contribution d'Alain Testart «Doit-on parler de "sacrifice" à propos des morts d'accompagnement? " (in Albert, 2005 : 34-57). Pour le dire rapidement, Testart nie toute dimension sacrificielle et donc religieuse à ce qu'il appelle les morts d'accompagnement, et qui sont des proches, des fidèles de grands personnages comme des rois et des chefs qui étaient mis à mort avec la déposition du défunt dans sa tombe et enfermés dans sa chambre sépulcrale ou tout près. En revanche, il reconnaît une dimension explicitement religieuse à certaines pratiques, y compris avec mises à mort d'hommes qui, une fois la tombe fermée, pouvaient avoir lieu en général autour ou à l'extérieur de la tombe. Je crois que Testart a raison de distinguer ces deux types de comportement, mais qu'il a tort d'y voir une différence, comme il dit «radicale» (ibid.: 40). Je pense au contraire, que ce sont des comportements qui se différencient, mais à partir d'une racine commune.

Il suggère que les morts d'accompagnement se justifient par la proximité de ces personnes avec le grand personnage. Elles lui appartiennent. «La raison de les enterrer est qu'elles sont la propriété du défunt» (ibid.: 49), disait un informateur. Il pense donc que la persistance d'un lien de propriété suffit à expliquer ces actes, et donc s'il s'agit de propriété, il n'y a rien de religieux. On pourrait déjà contester cependant qu'il n'y ait rien de religieux dans la propriété. Durkheim pensait le contraire en constatant que les formes les plus archaïques de propriété connues concernaient toutes des tabous protégeant des biens ou des personnes appartenant aux dieux et aux rois. La propriété n'a peut-être pas commencé, bien laïquement, comme le pensait Locke, comme une émanation, une extériorisation des personnes ordinaires.

Mais il n'y a pas de doute que les deuxièmes comportements sont des sacrifices religieux au sens le plus classique que retient Testart comme définition: une offrande rituelle à une divinité. Il ajoute:

Le fait que la chose soit détruite (que l'animal soit tué si c'est un animal) caractérise le sacrifice et le différencie d'une simple offrande: mais l'idée d'offrande est essentielle dans les deux cas. Il n'est pas de sacrifice qui ne soit offert, en principe à une puissance surnaturelle. Il y faut un tiers, en plus du sacrifiant et du sacrifié. Tout sacrifice suppose une translation, celle qui fait passer le sacrifié des mains du sacrifiant vers le destinataire du sacrifice. Or c'est ce qui manque dans le phénomène de l'accompagnement. Le roi scythe emporte avec lui son échanson, son palefrenier, sa concubine dans la tombe: ils étaient à lui (dans une sorte d'appartenance) durant sa vie, et le seront encore après sa mort. Ce qui était à lui reste à lui. C'est le contraire du transfert, le contraire d'une offrande: c'est plutôt le fait égoïste de celui qui dispose de gens et veut les garder après de lui toute l'éternité (ibid.: 41).

Ce raisonnement me parait contestable sur deux points. Il donne une définition classique, religieuse et restreinte du sacrifice comme d'une offrande à une entité surnaturelle. Par conséquent, les morts d'accompagnement appartenant déjà au mort ne peuvent pas lui être sacrifiés, c'est-à-dire donnés. Il n'y a donc rien de religieux dans les morts d'accompagnements, mais 
seulement dans les sacrifices faits une fois la tombe fermée. Cette définition du sacrifice a le mérite d'être précise, parce qu'elle est restreinte et conforme à nos usages d'inspiration chrétienne. Mais elle me parait aussi de ce fait ethnocentrique. Elle suppose que le sacrifice est toujours et partout une offrande positive, c'est-à-dire destinée à la conjonction avec le destinataire, la création d'un lien avec celui auquel on donne, ce qui est bien le cas du sacrifice chrétien donné à un Dieu, du moins supposé bon. Car l'offrande peut avoir un tout autre sens, quand on craint et redoute celui auquel elle est faite: elle vise la disjonction, on lui donne pour n'avoir plus à être en relation avec lui, ou qu'il ne se fâche pas contre nous, qu'il ne revienne pas, qu'il nous lâche les baskets dirait-on dans un style peu académique, surtout si c'est un mort et un mort puissant qui peut tourmenter les vivants. Et c'est bien ce que dit le Mwila cité par Testart: «Ils disent que la raison de les enterrer est qu'elles sont la propriété du défunt et que si elles ne l'étaient pas [enterrées], son fantôme serait très en colère et viendrait causer du tort aux vivants " (ibid.: 49). Testart retient la première affirmation et laissse tomber la seconde partie. Ce qu'il ne voit pas, c'est l'ambiguïté du don, ce que j'appelle la "pharmakologie du don »: ambiguiité de la chose donnée quand un don peut véhiculer un remède et un poison, ambiguiité du donataire quand il est désiré et craint, ambiguité des donateurs selon qu'ils veulent se séparer et en même temps s'unir au donataire. Et il ne voit pas ce problème pharmakologique, parce qu'il pense que l'idée d'offrande est plus importante dans la définition du sacrifice que le fait de mettre à mort ou de détruire ce qui est offert. La question est donc bien du statut et des raisons de la négativité que représente l'acte de détruire. L'acte de détruire est second par rapport à celui de donner quand le donateur veut une relation conjonctive avec le donataire, mais il est premier par rapport à l'acte d'offrir quand il veut une relation disjonctive. En réalité, la totalité du processus sacrificiel est irréductible au don parce qu'il détruit, et il détruit parce qu'il veut obtenir une disjonction : le sacrifice est ce qui opère la conjonction entre les hommes en les disjoignant d'une menace. Les morts d'accompagnement ont une dimension sacrificielle, car il s'agit de séparer le chef mort avec tout ce qui lui appartient d'avec les vivants, de mettre une distance entre lui et ceux qui restent, et cette séparation, cette rupture est la condition d'une relation apaisée avec lui. Le sacrifice est un acte symbolique et comme tel humain: il vise comme tout acte symbolique, tout acte de pensée donc, tout rite (voir les rites de passages), à "séparer pour unir ", mais sa spécificité est qu'il le fait en détruisant, c'est la négativité imposée à la victime comme négation de la négativité de l'invisible, de la menace. Et c'est en cela qu'il n'est pas une offrande ordinaire, un don positif commun. Cette définition du sacrifice est à la fois plus large et plus précise que celle de Testart qui, empruntée au dictionnaire, vient du sens commun et reste curieusement théologique et occidentalo-chrétienne. Un sociologue durkheimien se serait méfié et n'aurait pas repris telle quelle cette définition qui nous est coutumière.

Ce qui fait la différence du sacrifice et du don «normal », c'est la destruction. Pour Testart, la destruction vient après l'offrande. Elle est secondaire. 
C'est faux. Car la destruction ne s'ajoute pas au don, comme quand on mange ensemble, elle le précède. Elle est donc plus essentielle. Elle sépare d'abord, comme le sacré, et réunit ensuite par les échanges. Dans ce contexte, la destruction est toujours la négation d'une négation, c'est la négation d'une négativité, d'un mal, d'un danger, d'une menace. Elle vise donc à créer une distance. Il faut donc bien distinguer les dons qui veulent tenir à distance ou annuler une négativité indésirable et les dons qui se passent dans un cadre où le problème de la distance est a priori réglé et supposent un contrat. C'est la différence, entre les humains, qu'a vue Mauss entre le don agonistique, où la distance n'est pas réglée d'avance, et les prestations totales. Dans le don agonistique, il y a négation de la négativité, car le donateur ne veut pas subir la domination du donataire. Il doit neutraliser sa volonté de domination. Il y a aussi le risque du don empoisonné, qu'il faut tenir à distance, au moins tant que son ambiguïté pharmakologique (remède, poison ?) n'est pas levée, ce qui est une des fonctions du rite. C'est pour cette raison qu'on ne doit pas trop séparer la forme et le contenu des rites et surtout des sacrifices. On ne peut comprendre les sacrifices hors de la problématique pharmakologique qui se demande aussi ce qu'ils refoulent. Le sacré (dangereux ou bienfaisant) et le profane (neutre), le pur et l'impur, l'ordinaire et l'extraordinaire, le faste et le néfaste, avec des différences importantes et dans des champs d'expérience différents, sont des systèmes élémentaires de valeurs, qui règlent ce avec quoi on peut s'unir, mais autant, ce dont il faut se séparer. Ce sont des pharmakologies culturelles, donc particulières, dans la suite d'une logique universelle qui fait préférer le remède au poison et la santé à la maladie. Pratiquement, l'art du rituel en général et du sacrifice en particulier est d'opérer une conjonction entre des hommes par une disjonction qui refoule ce qui est cru négatif, dangereux, malfaisant, pour obtenir la conjonction avec ce qui est cru positif, ou même l'ingérer s'il est transformé positivement par le rituel, comme dans le cas de la cuisine. Comme la cuisine, le rituel rend consommables des combinaisons pharmakologiques qui sans elle seraient empoisonnées. Je crois que la pharmakologie est le noyau actif des rituels, des systèmes de valeurs et, dans nos sociétés, des idéologies et des utopies. Les formes les plus intellectualisées de la pharmakologie se retrouvent dans cette partie la plus aiguë des grandes métaphysiques que Leibniz appelait les théodicées, qui sont en fait aussi des kakodicées. Elles ont étonné et fasciné Weber. Elles sont devenues une des parties les plus célèbres de sa sociologie de la religion, sans qu'il voit qu'elles venaient sans doute des plus obscurs rituels. Ce n'est pas la moindre ruse de la rationalisation et de l'intellectualisation.

\section{Postface}

Je remercie d'autant plus les Archives de sciences sociales des religions de me donner l'occasion de réagir aux textes de la rencontre de Toulouse qu'ainsi elles me permettent de continuer un dialogue auquel, en mon absence, je n'avais pris part que par l'intervention précédente. 


\section{Contester l'opinion dominante}

"Je dois admettre que si je m'accorde largement avec l'enquête généalogique de Tarot, comme je l'ai dit, sur la dévalorisation du rite dans la pensée occidentale rationaliste et sur la nécessité de repenser entièrement la place du rite dans nos sociétés, je ne vois pas trop ce qu'apporte le détour par Girard ». Ce jugement de Christophe Grellard sur Girard est d'autant plus intéressant ou important qu'il n'est sûrement pas l'exclusivité de son auteur, car il a pour lui l'opinion dominante aujourd'hui encore de la majorité des anthropologues, des sociologues et des philosophes. Il y a toujours un problème de la réception en France de cette œuvre, pour de bonnes et de moins bonnes raisons.

Du côté des bonnes raisons et des plus sérieuses, l'œuvre de Girard est ambiguë du point de vue épistémologique et elle l'est doublement. C'est un «littéraire» qui, dans la Violence et le Sacré, s'est aventuré dans le domaine de l'anthropologie et de l'ethnologie, sans expérience de terrain. Mais une fois qu'il arpente ce domaine qui n'est pas le sien, il semble l'abandonner dans Des choses cachées depuis l'origine du monde pour celui qui parait lui être le plus opposé, la théologie, et pire, l'apologétique, en se mettant au service de la cause parfois dite "judéo-chrétienne". Il contrevient donc doublement aux règles élémentaires de la rigueur épistémologique par le mélange des disciplines et plus gravement en enfreignant, par un choix partisan, la neutralité axiologique et donc ce "regard éloigné ", qui est à la base des sciences sociales en général et de l'anthropologie en particulier, mais qu'il troque pour un regard engagé.

Néanmoins, l'œuvre de Girard aurait eu sa place, pour cette raison même, dans ce colloque consacré à la relation de la théologie et de l'anthropologie comme un cas éminent du passage de l'une à l'autre. Dans les termes de JeanPierre Albert, il ne s'agirait pas seulement d'un cas de "proximité inavouée " entre théologie et anthropologie, mais bel et bien avouée et revendiquée, ce qui la rend aussi provocante qu'un nez dans un visage. C'est probablement une des raisons pour lesquelles on évite de discuter avec cette œuvre. Comme pour l'adultère, elle aurait été mieux acceptée si elle avait gardé clandestine cette liaison dangereuse ou inavouable! Donc, pour reprendre les catégories employées par J.-P. Albert, on pourrait se servir de cette œuvre pour démontrer la thèse qu'il défend, que l'approche emic, celle du croyant, de l'adepte et du théologien peut faire un bon bout de chemin vers l'anthropologie, vers le point de vue etic, pris au triple sens de réunir la capacité de distanciation, la comparaison interreligieuse ou interculturelle et la construction systématique des faits qui ne sont pas le souci des croyants en général, mais parfois des théologiens et sûrement de tous les anthropologues, et de Girard lui-même. Au point que bien des ethnologues, légitimement soucieux d'abord de la complexité empirique des systèmes si divers qu'ils rencontrent, ne cessent de lui reprocher le côté systématique de ses positions au point de dénoncer un nouveau dogmatisme. 
Mais je dois avouer que ce n'est pas d'abord dans cette perspective de critique épistémologique que j'ai introduit Girard dans mon papier, même si pour ma part, je pense, dans la tradition wébérienne, que si la neutralité axiologique n'est pas une condition normale de l'existence ou une attitude naturelle de l'esprit, c'est une condition sine qua non des sciences sociales, du moins pendant le temps de leur exercice. Elle répond à l'impératif de suspendre (épochê) ses propres jugements de valeur, au moins pendant l'effort de comprendre des points de vue dont les valeurs peuvent être en contradiction avec celles de l'observateur ${ }^{1}$. $\mathrm{Au}$ fond, le problème n'est pas de savoir si Girard ou n'importe quel social scientist est chrétien ou marxiste ou agnostique, ou autre chose encore, mais de constater et d'évaluer en quoi ses convictions intimes joueraient comme une aide, une facilitation ou au contraire comme un obstacle à la compréhension du comportement des Autres, de ceux qu'il étudie. L'objectif des sciences sociales est de comprendre et d'expliquer en les recontextualisant les motivations des acteurs et la logique de leur action, qu'on les partage ou pas. Ceci suppose que l'interprète ait assez de distance pour jouer avec l'univers de sens et de valeurs auquel il adhère personnellement sans le laisser s'interposer spontanément ou automatiquement comme un filtre imposé et une raison supposée légitime a priori de défendre ou de refuser les comportements et les valeurs de l'autre. La neutralité axiologique vise à neutraliser les effets d'adhésion ou de rejet a priori, qui découlent si souvent des adhérences identitaires, des processus d'identification personnelle et collective, qui sont au minimum préconscients. Elle veut dire non pas que l'interprète doit être systématiquement pour son compte un incroyant ou un agnostique - ce que tout le monde est à l'égard de tel ou tel système de pensée -, mais que dans l'exercice de compréhension de l'Autre qui est sa tâche spécifique, il est un traducteur de faits qu'il doit respecter puisqu'ils sont donnés, non un juge qui les autorise ou les interdit, ou un témoin qui les défend ou les dénie.

Mais il est vrai que cette démarche interprétative expose les théories, surtout naissantes, produites par les sciences sociales, aux risques de la circularité, bien soulignés par C. Grellard. En effet, elles commencent toujours par une sélection de faits jugés décisifs en fonction de critères qui reposent initialement sur un cercle herméneutique. La seule façon d'y échapper, me semble-t-il, est de poursuivre contradictoirement l'enquête pour voir si la théorie est féconde, si elle se révèle apte ou non à intégrer une quantité croissante de faits nouveaux, comme il est arrivé avec la théorie de l'évolution des espèces qui a commencé par avoir beaucoup plus de faits qui jouaient contre elle qu'en sa faveur. La même chose arrivera peut-être aux spéculations de Girard, si du moins on met la main à la pâte.

1. Pour le dernier état de mes réflexions, par exemple sur la neutralité axiologique (voir index) je me permets de renvoyer une fois pour toutes à Tarot, 2019. Dans cet ouvrage, trois bévues sont à corriger: p. 19, il s'agit non de Jacques Testart mais bien de l'ethnologue, le regretté Alain Testart; p. 125, Judith Schlanger n'est pas américaine mais franco-israélienne et son ouvrage cité n'a pas eu besoin d'être traduit en français; enfin p. 468 note 1, il s'agit de Michel et non de Michèle Fromager. 
Ma raison d'introduire Girard dans le débat était d'abord historique. Lorsqu'il a publié la Violence et le sacré en 1972, à la fin de l'époque où le structuralisme de Lévi-Strauss ${ }^{2}$ régnait presque sans partage dans les milieux intellectuels et universitaires, il a eu le mérite d'engager une critique de sa pensée et de son hégémonie si bien accordée à l'air d'une époque de déclin et de sécularisation du phénomène religieux. Il a osé recentrer le phénomène religieux autour de ses deux pôles les moins vus ou les plus méconnus ou les plus scandaleux pour le structuralisme en particulier et pour notre culture moderne en général, et donc même pour les théologiens qui en participent peu ou prou: les rites et, dans les rites, les sacrifices et dans les sacrifices, les sacrifices humains. Reconnaître les manques des positions de Lévi-Strauss sur la religion et le sacré qu'il congédie comme des catégories inutiles ou infondées ${ }^{3}$ ne signifie certes pas qu'on dénie son immense apport à l'analyse des mythes. L'idée que la planète des mythes est ronde ou qu'un mythe est composé de toutes ses versions est toujours féconde ${ }^{4}$. Les Mythologiques appartiennent aux monuments de la pensée du $\mathrm{xx}^{\mathrm{e}}$ siècle. Mais ce gain de la méthode structurale a un coût, elle exclut toute démarche généalogique ou sociogénétique et elle évite l'analyse de la violence et du désordre, puisqu'elle focalise d'emblée sur la recherche des structures à la base de l'ordre considéré comme stable et acquis.

C'est donc d'abord au titre de symptôme d'un effort de réévaluation des rites, après un long dédain, que j'ai convoqué Girard. Il suffira de rappeler ses thèses principales, même si elles sont très générales, pour donner les raisons de mon "détour ". La première est le primat du rite sur le mythe ${ }^{5}$ et son autonomie performative: les rites forment un monde qui a son mode de fonctionnement propre. Ils sont à l'origine de la formalisation des institutions. Cette thèse initiale me parait la plus importante et pour moi conclut un débat en serpent de mer qui court depuis la naissance des sciences des religions. La deuxième thèse fondamentale de Girard, encore difficile, est que dans le foisonnement des rites et leur généalogie, il faut donner la première place, du point de vue institutionnel, aux sacrifices. Ce n'est pas une évidence. Pourquoi pas aux rites de passage? La troisième thèse, qui explique et justifie grandement la précédente, c'est que les sacrifices ont d'abord rapport à la violence entre et

2. Il y a structuralisme et structuralisme! Celui qu'on attribue aussi à Dumézil prête, à juste titre, la même attention aux rites qu'aux mythes, mais son influence fut autrement plus restreinte à des milieux spécialisés. Voir Tarot, 2008: 521-522, 526-527.

3. J'ai montré (Tarot, 2008: 378-384; Tarot, 2019: 73-74) comment Lévi-Strauss fonde cette exclusion sur une lecture très particulière qui se sert de Mauss pour exclure la sociologie de la religion de Durkheim.

4. Elle a été encore récemment appliquée par exemple aux vies du Bouddha (Faure, 2018).

5. Comme le montrent aussi les religions qui ont perdu grandement leur mythologie mais gardé leur système rituel, comme en Chine (Tarot, 2019: 115-117) ou la religion romaine (ibid.: 66-67). En écho aux exemples donnés par Grellard, on peut ajouter la maxime des mimânsaka hindous si paradoxale du point de vue occidental, selon laquelle «il faut sacrifier même si les dieux n'existent pas". 
sur les hommes, parce que le sacré, et bien qu'il puisse s'étendre a tout ou presque - toute chose pouvant être sacralisée, comme l'ont montré usque ad nauseam les morphologies du sacré - a d'abord rapport à la violence par la séparation de la bonne et de la mauvaise violence. Ces trois thèses, si elles sont exactes et encore une fois, malgré leur généralité, suffiraient à fonder la légitimité d'une ritologie en soi, et donneraient un contenu à ce "gros mot". Donc si le philosophe, et pas seulement lui d'ailleurs, pense à juste titre que le « rite est un objet dont la richesse mérite qu'il s'en empare» (Grellard), il me semble avoir intérêt à s'emparer aussi, de façon certes critique, des intuitions girardiennes, au moins pour les discuter.

\section{Points de convergence et difficultés persistantes}

Nonobstant cette divergence sur l'appréciation de l'œuvre de Girard, on peut relever des points de convergences entre les quatre papiers. En voici quelques-uns.

La ritologie n'est pas encore un champ d'étude spécifique constitué, mais elle mériterait tout à fait de le devenir (Rauwel). Les rites ont été moins étudiés, car jugés avec un certain mépris ${ }^{6}$ (Rauwel, Tarot). Ils ont été renvoyés comme rites des autres dans l'altérité du primitivisme, de l'exotique, de l'irrationnel, du prémoderne (Rauwel, Tarot).

Ces phénomènes de dévaluation ou d'incompréhension tiennent de près au christianisme dès sa naissance, en réaction au judaïsme, à sa critique du légalisme et du pharisaïsme, à sa jonction avec la philosophie grecque (Rauwel, Tarot). Bien que le Moyen Âge ait été une époque d'emprise rituelle considérable, «il est certain que certains aspects de la théologie médiévale jouent un rôle clé dans l'oubli du rite, lequel est significatif du passage à la modernité » (Grellard; Rauwel, Tarot), souvent polarisée par l'idéal (ou l'utopie ?) d'une "religion sans ritualité » qui paraissait le terme de l'évolution religieuse de l'humanité.

De ce point de vue, l'époque moderne récente est bien l'héritière d'une tradition intellectuelle chrétienne qu'elle a radicalisée. Avec la Réforme, Spinoza et les romantiques, la critique du rite se fait aussi bien au nom des droits de la rationalité impersonnelle que de ceux du sentiment, de l'expérience vécue, de la spontanéité subjective individuelle dans la tradition romantique toujours vivante aujourd'hui, par exemple dans le New Âge.

Car les systèmes rituels vont de pair avec l' "obligation rituelle» d'être suivis et imités scrupuleusement à l'image de ce qui s'est toujours fait. "C'est la coutume» (Grellard, Albert, Tarot). Ils se revendiquent donc comme antiques ou hors d'âge (Rauwel). Or, ils reprennent dans leurs séquences des gestes et des pratiques, des objets ordinaires et de la vie quotidienne. "Les séquences rituelles sont souvent des reprises d'actions et de chaines opératoires de

6. Mépris qui a pesé lourd sur l'histoire des religions, comme le montre bien Scheid, 2019: 7-37, version remaniée d'un texte composé avec Jean-Louis Durand (Scheid, Durand, 1994). 
la vie quotidienne: faire un don, partager de la nourriture, solliciter une autorité supérieure, etc.» (Albert). Mais vient un moment où ils butent sur du «mystère ", amènent à de l'extraordinaire, à de l'indicible au point qu' "une dimension d'inintelligibilité structurelle est nécessaire au rituel» (Rauwel). C'est aussi ce que constate la définition de la ritualité par l' "anomalie pragmatique » (Albert). Elle est certes particulièrement sensible, d'un point de vue etic, dans notre société sécularisée qui choisit systématiquement la technique contre le rite, car la technologie y est devenue le modèle de la rationalité instrumentale en acte et une condition majeure de la puissance. Toutefois, d'un point de vue emic, l'anomalie pragmatique est-elle ressentie comme telle par le jardinier trobriandais des Jardins de corail? Il mêle les techniques magiques à celles du jardinage dont il a une connaissance pragmatique. Et non sans raison : il cherche à mettre toutes les chances de succès de son côté. Il reste que les rites comme le phénomène religieux en général renvoient à de l'extraordinaire et à de l'extraquotidien, qu'ils sont contraints non seulement de traduire, mais d'appliquer dans le langage et le monde de la vie ordinaire et quotidienne.

C'est que le rite, comme tout phénomène religieux, renvoie ultimement à l'exceptionnel: en termes wébériens ${ }^{7}$ à l'extraordinaire et à l'extraquotidien, en termes durkheimiens au sacré et à l'effervescence, en termes girardiens à la violence fondatrice. Mais l'ensemble des rites ne transforment la vie des adeptes que s'ils se quotidianisent, si leur pratique ordinaire réussit à médiatiser quelque chose de leur efficace puisée dans l'exceptionnel. La quotidianisation est donc l'épreuve du succès d'une religion avant de devenir possiblement sa tombe si la religion ne peut plus entretenir et soutenir ces dialectiques.

On conviendra donc qu'une ritologie doit travailler son objet dans deux directions différentes. Celle d' "une ritologie "totale" [qui] inclut aussi bien le discours $d u$ rite que le discours sur le rite» (Rauwel). On remarquera ici l'analogie de cette exigence avec la méthode structurale pour laquelle un mythe est fait de l'ensemble de ses versions ou variations dont participent ses exégèses. L'autre voie, en sens inverse, cherche à suivre non les adaptations, réinterprétations, substitutions, bref l'inventivité proliférante des rituels, mais au contraire à remonter vers le «noyau dur rituel» (Rauwel). Ce qui est la voie d'une archéologie en quête des fondamentaux les plus pérennes et qui donc distingue l'archaïque du primitif ${ }^{8}$. La différence de ces deux approches reparait à la fin de la synthèse de C. Grellard, quand, dans la ligne de l'analyse régressive, il défend sa thèse fonctionnaliste du rite qui assure d'abord la cohésion sociale, même indépendamment de la croyance aux êtres surnaturels et aux mythes, qui, au contraire, sont inhérents au religieux pour Jean-Pierre Albert, et qui fournissent un peu partout dans le monde le point de départ des

\footnotetext{
7. Sur ces deux oppositions wébériennes du quotidien à l'extraquotidien et de l'ordinaire à l'extraordinaire que je crois analogues en partie à celle de Durkheim entre le profane et le sacré, et dont elles tiennent lieu au moins fonctionnellement dans la religiologie du sociologue allemand, voir Tarot, 2019: 90-94.

8. Sur cette distinction essentielle, voir Tarot, 2019: 36-37.
} 
spéculations de l' " anthropologue théologien ». Toutefois, J.-P. Albert cherche aussi le noyau dur sinon des rituels, du moins des sacrifices dans un ensemble de traits, peut-être pas constants, mais récurrents: 1. La ritualité, 2 La dette et l'obligation. 3. La conservation de l'ordre du monde. 4. La manipulation du négatif. 5. La présence d'anomalies par rapport à l'idée intuitive de justice.

En fait, il n'y a pas à choisir, puisque les deux voies existent bien dans les faits et sont également légitimes, même si on ne peut les parcourir en même temps puisqu'elles vont en sens contraire! Elles mènent en des sens opposés vers les territoires de ce que j'ai appelé la religion par en haut et la religion par en bas ${ }^{9}$. La religion définie par J.-P. Albert comme « les rapports entre les hommes et cette réalité supérieure ", comprise comme surnaturel ou substance spirituelle, relève en partie de la religion par en haut. Alors que C. Grellard, à travers la cohésion sociale, pointe vers la religion par en bas, souvent infrareligieuse par rapport aux normes et contenus de la précédente. On voit qu'elles ne sont pas de même niveau socio-anthropologique. La seconde conduit à l'analyse de comportements qui ultimement relèvent de la meute ou de la bande et qu'on retrouve dans les mécanismes les plus frustes comme ceux du bouc émissaire. La première débouche ultimement sur les questions de la métaphysique, de l'ontologie, de la théodicée, du fondement des valeurs, de la spiritualité ${ }^{10}$ dans tous les sens du mot, etc.

D’où l'importance de voir les métareprésentations, fussent-elles estompées, qui opèrent ou ont opéré comme des vecteurs entre les deux. On essaiera de le montrer pour finir en reprenant les exemples bibliques introduits par J.-P. Albert et qui confirment dans un constat de saveur fort girardienne, qu' «au total, le sacrifice semble hanter les textes du Pentateuque bien plus qu'on aurait pu le penser. Et cela est encore plus vrai si l'on prend en compte certains épisodes qui, sans être désignés comme tels, entrent en fait dans une logique sacrificielle» (Albert).

\section{Confirmation et prolongements}

Girard et Lévi-Strauss sont bien convaincus que les cultures humaines sont des systèmes de différences, fondées sur des oppositions finalement binaires, produites par l'entendement humain et capables de fonctionner dans tous les registres de l'expérience, de l'expérience sensible à l'expérience mystique (le haut et le bas, le chaud et le froid, le cuit et le cru, le pur et l'impur, le vrai et le faux, le bien et le mal, le permis et le défendu, le sacré et le profane, le caché et l'apparent, etc.). Girard ${ }^{11}$ appelle «crise sacrificielle» les moments de décomposition, d'érosion des différences et de rupture ou de perte du consensus sur ces systèmes des différences et leurs hiérarchies. Il en résulte une montée de la rivalité pour imposer un autre ordre ou restaurer l'ancien et expulser les

9. Sur cette distinction voir, Tarot, 2018: 58-61.

10. Sur les variations de sens de ce mot, Tarot, 2019: 468-469.

11. Pour une présentation plus détaillée, voir Tarot, 2019: 75-76. 
opposants ou les rivaux. Un groupe stressé par ces luttes et cette instabilité de la violence alternée, mimétique, est tenté d'en sortir en trouvant un bouc émissaire, pris en son sein, dont l'expulsion ou la mise à mort refait l'unité du groupe en transformant la violence potentiellement de tous contre tous (c'est une représentation limite) en violence de tous contre au moins un, ce qui réintroduit un principe de différenciation qui refait une unanimité. L'ordre instauré ainsi grâce au bouc émissaire, celui-ci passe dans les mythes du statut de pharmakos et de pharmakon, c'est-à-dire de monstre et de poison, accusé d'être la cause de tous les maux du groupe, à celui de remède auquel le groupe doit son existence en tant que groupe. Il est alors idéalisé et donne naissance aux dieux. Les hommes croient n'être pour rien dans ce processus, dans cette violence et sa transformation, ce que Girard appelle la méconnaissance. Tout est l'œuvre des dieux, des démons ou des esprits. Mais leurs dieux ou leurs esprits, ambigus et bivalents, portent la marque de l'ambivalence des hommes à l'égard de la violence. Ils réunissent les traits de puissances redoutables, dangereuses, menaçantes, colériques, qu'on ne doit approcher qu'avec des précautions, et de puissances bienveillantes, justicières et apaisantes, consolatrices, mais dont la médiation est toujours nécessaire à la vie sociale. Pour Girard, se tient donc au principe du sacré et de ses casuistiques rituelles, la réification, l'objectivation de la violence collective sous son double aspect de violence destructrice dont la menace pèse constamment sur le groupe, peut revenir à chaque instant, la violence qu'on dira négative, et de la violence positive, parce que fondatrice des différences essentielles à la base des institutions qui permettent d'échapper à la première. Dans cette perspective, le sacrifice rituel est le premier des rites et la première des institutions, car il permet, par le transfert de la violence collective d'un bouc émissaire à une victime de substitution, de rejouer à un niveau symbolique, la crise fondatrice chaque fois que la société est en danger et de réactualiser ce qui fait la fonction de tout rite en général et du sacrifice de façon éminente: assurer la conjonction, donc l'unité d'un groupe d'hommes par une disjonction, donc une expulsion, de ce qui passe pour la menacer. D'un point de vue etic, la métareprésentation la plus frappante de ce schéma est le sociocentrisme absolu du point de vue emic: l'ordre cosmique tient ou s'effondre avec l'ordre social interne du groupe.

Les hypothèses girardiennes forment un scénario construit à partir de quelques faits attestés, mais dont toutes les parties ne sont pas également démontrées ou démontrables ${ }^{12}$. C'est pourquoi ce scénario doit être complété dans ses parties obscures ou elliptiques. J'en donnerai quelques exemples. La culture est bien un système de différences. Mais ces différences ne sont pas seulement différentielles, comme l'ont dit les structuralistes, ou des oppositions pertinentes sur le modèle des phonèmes dans le système abstrait de la langue. Elles diffèrent pour les raisons complexes, mais existentielles, qui font que tout le monde préfère la santé à la maladie, la beauté à la laideur, la richesse

12. Pour plus de développements, voir Tarot, 2019: 77-83. 
à la pauvreté, etc. Le pur et l'impur ne servent pas qu'à une mise en ordre, ce n'est pas qu'un critère de rangement, comme le veut l'ouvrage classique de Mary Douglas. Ils sont discriminants, dans toute l'ambiguïté de ce mot. Le pur doit combattre et chasser l'impur contagieux ou sinon se protéger ou en être protégé. Le chaos n'est pas que le contraire de l'harmonie sociale ou du monde, mais son contradictoire. Ces oppositions mobilisent et polarisent non seulement des distinctions, mais des jugements de valeurs, des refoulements, des passions, des haines, des peurs, des angoisses. La négativité n'est pas qu'une négation, mais porte comme son horizon la menace de la mort ou de l'anéantissement, ce qui complique beaucoup le problème des valeurs et des conflits de valeur, qui ont vite fait de déraper du conflit logique au conflit guerrier. Cet imbroglio se retrouve au cœur de ce que j'appelle la pharmakologie du bouc émissaire, écrite avec un $\mathrm{k}$ pour rendre la différence du pharmakos, au masculin, qui est toujours une personne, un humain, par opposition à la pharmacologie des pharmaciens qui est une science exacte et quantitative étudiant les effets des pharmaka, au neutre, des substances toxiques sur les organismes sains ou malades. La pharmakologie n'est pas une science objective puisqu'elle repose sur des accusations que le mythe et l'idéologie justifient. Or ces accusations peuvent être vraies, fausses ou outrées (projectives). J'écris pharmac/kologie ${ }^{13}$, par une condensation imperceptible à l'oreille, pour attirer l'attention sur l'ambiguité des situations très nombreuses, de la sorcellerie au combat politique, des luttes religieuses aux conflits sociaux qui passent des substances, des idées ou des situations dites empoisonnées à l'accusation qui rend des hommes responsables de ces maux. Ce qui caractérise ce que j'appelle l'indifférenciation pharmacl kologique. Or les pharmac/kologies négatives de l'autre homme passent toujours par des métaphores monotones dans leur récurrence. Elles se ramènent à cinq grandes familles ou catégories: le poison, la maladie contagieuse, le criminel, l'animal, le monstre. Chaque religion combine une multiplicité de pharmac/kologies. Girard a mis a jour celle de la violence, que j'appelle pour cette raison bialogique. Mais il y a celle du pur et de l'impur qui plonge dans l'expérience du corps propre, celle du faste ou du néfaste au sens moderne qui se fonde sur les aléas de l'action liée à la chance ou à la malchance, celle du don qui peut être empoisonné, celle de la mémoire qui prescrit le souvenir ou l'oubli, celle du péché et du saint qui suppose une loi explicite, etc. Mais c'est la pharmac/kologie de la violence qui exerce l'hégémonie sur les autres avec lesquelles elle se recombine: la violence contre un «sang impur» est pure, plus pure ou licite, alors que la même violence contre un sang pur serait impure ou illicite, etc. Une pharmac/kologie associe toujours l'accusation d'un poison à la proposition d'un remède, ce qui a son incidence sur le rapport aux valeurs et à ce qui les contredit. Les phamarc/kologies se retrouvent, mais moins nombreuses et immanentisées, dans les idéologies modernes et les utopies dont elles constituent toujours le noyau productif.

13. Pour plus de détails sur la notion et les emboitements des pharmac/kologies, Tarot, 2019: 101-108. 
Mais, pour compléter le modèle girardien, il faudra aussi tester ses capacités à faire saillir les logiques sacrificielles en s'en servant comme d'un idéal-type confrontable à un nombre infini d'épisodes, de récits ou de rituels. Là où on a quelque raison de soupçonner leur présence, la comparaison, également attentive aux ressemblances et aux différences, de l'idéal-type avec les données, fera ressortir les logiques sacrificielles et leurs transformations. C'est d'autant plus utile que, comme le souligne J.-P. Albert, les sources bibliques, pour ne prendre qu'elles, sont très diversement explicites. Quoiqu'il en soit de l'historicité plus que douteuse de ce genre de récit, on peut dégager la logique de leurs métareprésentations, au moins ici sous forme d'esquisses à peine ébauchées. Dans le récit de Noé, pas de bouc émissaire ni de mise à mort d'un individu évidemment. On est très loin du récit princeps girardien, puisqu'il s'agit d'un récit d'alliance. Mais il contient plusieurs éléments conformes au modèle. D'abord, il fait suite à la pire crise sacrificielle imaginable, la plus universelle et la plus radicale puisque le déluge atteint toute l'humanité et l'ordre cosmique. Il confirme que l'ordre du monde et de la culture sont faits de différences, "froid et chaleur, été et hiver, jour et nuit» (Gen. 8, 22). Mais la nouveauté par rapport au schéma princeps, c'est que la violence est attribuée aux hommes, tandis que désormais Dieu s'engage à garantir indépendamment de la méchanceté humaine ces différences qui ne cesseront jamais. Noé, seul homme juste, apparaît en quelque sorte comme l'inversion du bouc émissaire. Mais il sauve la structure «tous contre un » en l'inversant en un contre tous, ce qui permet le retour de la différenciation, en inversant ses pôles pharmacl kologiques: de "tous victimes d'un bouc émissaire responsable de tout le mal» on passe à «un juste après tous les mauvais ». Il est très exact de dire que «Yahvé semble à présent s'accommoder de la méchanceté des hommes » et renonce à déclencher, à cause d'elle, un nouveau cataclysme qui ramènerait le monde au chaos. L'idée est donc que les hommes ont toujours le pouvoir de déclencher la crise sacrificielle qui annule les différences, mais que désormais l'ordre cosmique tient indépendamment de l'ordre social, ce qui constitue une "sécularisation" du monde par rapport au mythe, mais non à la théologie. Le sacrifice de Noé est central, comme attendu, dans cette refondation. Or il débouche sur une alliance où Dieu s’engage à limiter sa violence répressive ( «je ne frapperai plus tous les vivants comme je l'ai fait» (v. 21), ce qui montre bien la réification de la violence, attendue par le modèle girardien, présente dans le sacré divin même yahviste. Dieu est fait d'un côté colérique et d'un autre apaisant. Cet engagement vient après le sacrifice, qui est présenté comme un sacrifice déjà rituel, complet, parfait, puisqu'il ne comporte que des animaux purs, c'est pourquoi ce sacrifice est indéniablement un don non empoisonné, donc acceptable et accepté, puisqu'il en monte « un parfum apaisant» (v. 21). Il entre dans la «pharmac/kologie positive» du don, ce qui n'est pas le cas de tous les sacrifices, qui ne sont pas du tout ou pas d'abord des dons quand la victime et le(s) destinataire(s) sont mauvais et qu'il s'agit seulement de s'en débarrasser, ce qui est le cas de la sorcellerie, de certains rites magiques et des sacrifices apotropaïques, par exemple aux puissances infernales. Dans le récit 
du déluge, la violence comme mauvaise violence vient de tous les hommes et pas d'un bouc émissaire, et d'autre part de Dieu qui restreint la sienne, ce qui écorne la méconnaissance ou la déplace avec le sociomorphisme du mythe. Désormais, la tenue ou la chute de l'ordre naturel ne dépend plus mécaniquement de la méchanceté des hommes et la violence de la colère divine ne tombera plus automatiquement sur eux.

Les Lévites sont bien la tribu sainte, sacrée, donc mise à part d'Israël, par et pour leur fonction sacerdotale d'enseigner et d'offrir les sacrifices, si on en croit Dt 33, 9-10. "Ils ont gardé ta parole, ils veillent sur ton alliance, ils enseignent tes coutumes à Jacob, ta loi à Israël; ils présentent le parfum à tes narines, l'offrande totale sur ton autel». Mais l'institution, dit le même texte, plonge dans la violence, à la suite des événements de Massa (épreuve) et Mériba (querelle), de la querelle du veau d'or. Il y a bien crise sacrificielle, mais limitée cette fois à ce ramassis de fugitifs qui n'ont pas encore intériorisé la loi et qui sont l'amorce du peuple d'Israël. Elle va jusqu'au conflit ouvert entre les partisans du Seigneur et les tenants du veau d'or, quoi qu'on mette sous ce terme. Moïse arguant d'un oracle du Seigneur appelle les premiers à liquider ces derniers. 3000 tombèrent tués par les fils de Lévi qui n'épargnèrent pas leurs parents. Ce massacre reçoit la légitimation d'une violence qualifiante, si l'on peut dire, de la part de Moïse: «Recevez aujourd'hui, l'investiture de par le Seigneur, chacun au prix même de son fils et de son frère et qu'il vous accorde aujourd'hui bénédiction»(Ex. 32, 29). En quoi cet épisode dans lequel on verrait plutôt le prototype sinistre d'une guerre de religion est-il sacré et sacrificiel? Il est sacré pour les Lévites, puisqu'il les met à part des autres israélites. Mais il n'est pas un sacrifice don complet comme celui de Noé, car il n'est pas dit que les massacrés sont des offrandes agréées, pures. Ils sont seulement expulsés, éliminés comme des dangers. Pourtant, il y a comme un contre-don, car en retour de leur geste les Lévites se voient gratifiés de la baraka divine. Il y a donc création, sinon d'une alliance, du moins d'un lien spécial entre les lévites et Dieu. Ce que les lévites ont "donné », et qui leur vaut le contre-don de la baraka, c'est la rupture des liens de sang et d'alliance de la parenté, puisqu'ils n’ont pas épargné leurs parents. Don négatif en quelque sorte, mais hautement préférentiel pour Yahvé. La conjonction des Lévites entre eux, qui les distingue du reste du peuple et les rapproche de Dieu, a été obtenue par le choix d'une disjonction d'avec leur parenté «naturelle». Sous la violence du fait (insoutenable pour nous, du point de vue étic... et éthique), il y a comme l'amorce d'un sacrifice spirituel. On a donc bien au final un sacrifice comme facteur d'alliance, mais par la grâce de Dieu, pour ainsi dire, car anthropologiquement, le meurtre de la parenté n'est pas un sacrifice don, mais un sacrifice apotropaïque dans un conflit violent où l'adversaire n'est pas une victime valorisée, mais à éliminer. Au plus près du plus fruste du modèle girardien, il confirme qu'un groupe d'homme peut se réunir (conjonction) par la disjonction (ici le massacre) d'une part des leurs, considérés par eux comme une menace. Mais, ce sacrifice élémentaire est ratifié par Moïse et reconnu créer un lien spécial entre les Lévites et Dieu 
puisqu'ils reçoivent sa baraka en signe de reconnaissance, ce qui introduit ce sacrifice dans la sphère du contre-don, alors qu'il ne comportait pas de don, d'offrande. En effet, les 3000 victimes n'ont pas été offertes à Yahveh, comme dans un rituel de sacrifice humain, mais seulement éliminées. C'est l'acte par lequel les Lévites se sont séparés des leurs qui, comme un don, tient lieu de contrepartie à la bénédiction comme contre-don qui scelle la relation spéciale de la tribu sacrée à son Dieu.

On dira, non sans raison, que le modèle girardien éclaire les faits bibliques parce qu'il en provient grandement. Donc qu'on se meut dans un cercle. C'est indéniable. Et d'autant plus que Girard revendique d'être branché sur la veine biblique. Mais la réponse est qu'il faut poursuivre l'enquête et l'analyse, là et ailleurs. En attendant, ces deux exemples confirment que tous les sacrifices ne sont pas des dons, seulement certains d'entre eux. Car la plupart des sacrifices visent d'abord à éloigner une force hostile, à imposer une disjonction définitive ou temporaire. La problématique pharmac/kologique du poison à éliminer par un remède est antérieure à la fonction dorologique du sacrifice, comme système de don et de contre-don. Elle l'englobe et la limite. Si donc on veut que tous les sacrifices soient des dons, il faut reconnaître, contre notre idée dominante du don, que tous les dons ne visent pas à faire de la conjonction, ce qui est bien le cas des dons empoisonnés. Certains, sans être empoisonnés, visent bien à opérer une disjonction comme quand on donne à un importun dont on veut se défaire. Aussi, je ne dirai pas comme C. Grellard que «la croyance en des entités surnaturelles ne semble pas nécessaire à la pratique d'un rite ", mais seulement "la croyance en des entités surnaturelles bonnes ou positives ", car s'il n'y avait pas de maux ou de menaces dont l'humanité cherche à se débarrasser, il n'y aurait ni rites ni sacrifices.

En conclusion, on pourrait dire, en d'autres termes, que la conjonction entre les hommes obtenue par la disjonction d'avec ce qui passe pour les et la menacer constitue l'infrastructure sacrificielle, monotone et répétitive, de tous les systèmes rituels, donc le "noyau dur" que nous cherchions, alors que la conjonction avec les forces positives et l'alliance issue du don et du contre-don forment la superstructure des systèmes sacrificiels, ce par quoi ils se différencient beaucoup plus, car elle prolifère dans leurs mythologies, leurs théologies, leur idéalisme, leurs idéologies voire leurs utopies dont devrait rendre compte le (trop ?) vaste projet d'une "ritologie totale». Les sacrifices purement disjonctifs sont communs à la sorcellerie et à la religion par en bas, les sacrifices disjonctifs et conjonctifs par les dons et les contre-dons, les alliances qui en sortent, tirent, non sans peine, la religion par en bas vers la religion par en haut. On voit la différence du rapport aux destinataires dans le traitement purement destructeur et infamant ou au contraire valorisant réservé aux victimes.

Camille TAROT Centre d'étude et de recherche sur les risques et les vulnérabilités, université de Caen camille.tarot@orange.fr 


\section{Bibliographie}

Albert Jean-Pierre (dir.), 2005, Sacrifice humain en Égypte ancienne et ailleurs, Paris, Soleb. Albert Jean-Pierre, Piette Albert, 2010, "Rite (Rituel, ritualité)» in R. Azria, D. Hervieu-Léger (dir.), Dictionnaire des faits religieux, Paris, Presses universitaires de France, p. 1102-1109.

Amselle Jean-Loup, 2013, Psychotropiques. La fièvre de l'ayahuasca en forêt amazonienne, Paris, Albin Michel.

FAure Bernard, 2018, Mille et une vies du Bouddha, Paris, Éditions du Seuil.

Kippenberg Hans, Riesebrodt Martin (eds.), 2001, Max Webers “Religionsytematik”, Tübingen, Mohr Siebeck.

SCHEID John, Durand Jean-Louis, 1994, " "Rites” et "Religion”. Remarques sur certains préjugés des historiens de la religion des Grecs et des Romains ", Archives de sciences sociales des religions, 85 , p. 23-43

SCHEID John, 2019, Rites et religion à Rome, Paris, CNRS Éditions.

ТАROT Camille, 2008, Le symbolique et le sacré. Théories de la religion, Paris, La Découverte. -, 2019, L'actualité de la religion. Introduction critique aux sciences sociales des religions, préface de Danièle Hervieu-Léger, Larmont, Le Bord de l'eau. 